have recently became commercially available for noninvasive measurements [3]. These developments can be helpful to further explore the role of neonatal lung function in the development of respiratory diseases in later life.

@ERSpublications

Impaired neonatal lung function is associated with life respiratory symptoms; its measurement at birth is important http://ow.ly/rJjyd

Anne C. van der Gugten ${ }^{1}$, Cornelis K. van der Ent $^{1}$ and Cuno S.P.M. Uiterwaal ${ }^{2}$

${ }^{1}$ Dept of Paediatric Pulmonology, Wilhelmina Children's Hospital, University Medical Center Utrecht, Utrecht, and

${ }^{2}$ Julius Center for Health Sciences and Primary Care, University Medical Center Utrecht, Utrecht, The Netherlands.

Correspondence: A.C. van der Gugten, Dept of Paediatric Pulmonology, Wilhelmina Children's Hospital, University Medical Center Utrecht, Office KH.01.419.0, PO Box 85090, 3508 AB Utrecht, The Netherlands.

E-mail: a.c.vandergugten@umcutrecht.nl

Received: Oct 022013 | Accepted: Oct 032013

Conflict of interest: Disclosures can be found alongside the online version of this article at www.erj.ersjournals.com

\title{
References
}

van der Gugten AC, Uiterwaal CS, van Putte-Katier N, et al. Reduced neonatal lung function and wheezing illnesses during the first 5 years of life. Eur Respir J 2013; 42: 107-115.

2 Collins SA, Pike KC, Inskip HM, et al. Validation of novel wheeze phenotypes using longitudinal airway function and atopic sensitization data in the first 6 years of life: evidence from the Southampton women's survey. Pediatr Pulmonol 2013; 48: 683-692.

3 MediSpirit. www.medispirit.eu Date last accessed: November 18, 2013

\section{Bronchoalveolar lavage cytology resembles sarcoidosis in a subgroup of granulomatous CVID}

To the Editor:

We read with great interest the article by BOUvRY et al. [1] regarding similarities and differences between interstitial lung disease (ILD) in granulomatous common variable immunodeficiency (CVID) and sarcoidosis. In this retrospective study, differential bronchoalveolar lavage (BAL) cytology was analysed in 14 patients with granulomatous CVID and ILD. The authors found BAL lymphocytosis $(>20 \%)$ in 11 out of 14 patients and a mean \pm SD proportion of BAL lymphocytes of $37.3 \pm 15.3 \%$. Unlike the sarcoidosis group $(5.3 \pm 4.0)$, the CD4/CD8 ratio was low in the analysed patients with granulomatous CVID and ILD $(1.6 \pm 1.1, n=10)$ and even $<1$ in half of the patients $(n=5)$. Bouvry et al. [1] concluded that there are significant differences in differential BAL cytology between sarcoidosis and granulomatous CVID.

We therefore retrospectively analysed a subgroup of 11 CVID patients (seven females and four males) with histologically proven granulomatous disease according to the inclusion criteria used by BouvRY et al. [1] and analysed BAL findings. Patients were $41.5 \pm 15.2$ years of age and referred to the Centre of Chronic Immunodeficiency, University Medical Centre Freiburg (Freiburg, Germany) between 2003 and 2012. CVID was diagnosed based on the European Society for Immunodeficiencies/Pan-American Group for Immunodeficiency criteria [2]. In nine out of 11 patients, transbronchial biopsy was performed during bronchoscopy. In six (67\%) of the biopsies, lymphocytic infiltrations could be detected, which did not fulfil the criteria for lymphocytic interstitial pneumonia. BAL lymphocytes $(53.3 \pm 19.8 \%)$ exceeded $20 \%$ in all patients. CD4 cells accounted for $68.7 \pm 18.1 \%$ and CD8 cells for $16.6 \pm 8.2 \%$ of BAL cells. In contrast to the results reported by BOUVRY et al. [1], we found a high CD4/CD8 ratio of $6.8 \pm 7.0$ and no patient had a $\mathrm{CD} 4 / \mathrm{CD} 8$ ratio $<1.5$. Moreover, the $\mathrm{CD} 4 / \mathrm{CD} 8$ ratio $(\mathrm{n}=10 ; \mathrm{r}=0.719, \mathrm{p}=0.019)$ and the percentage of $\mathrm{BAL}$ CD4 lymphocytes $(n=9 ; r=0.816, p=0.004)$ correlated negatively with BAL neutrophils $(6.5 \pm 8.5 \%)$. The study by BOUvry et al. [1] showed significant differences in chest high-resolution computed tomography (HRCT) morphology between CVID and sarcoidosis patients. In particular nodules, air bronchograms, halo 


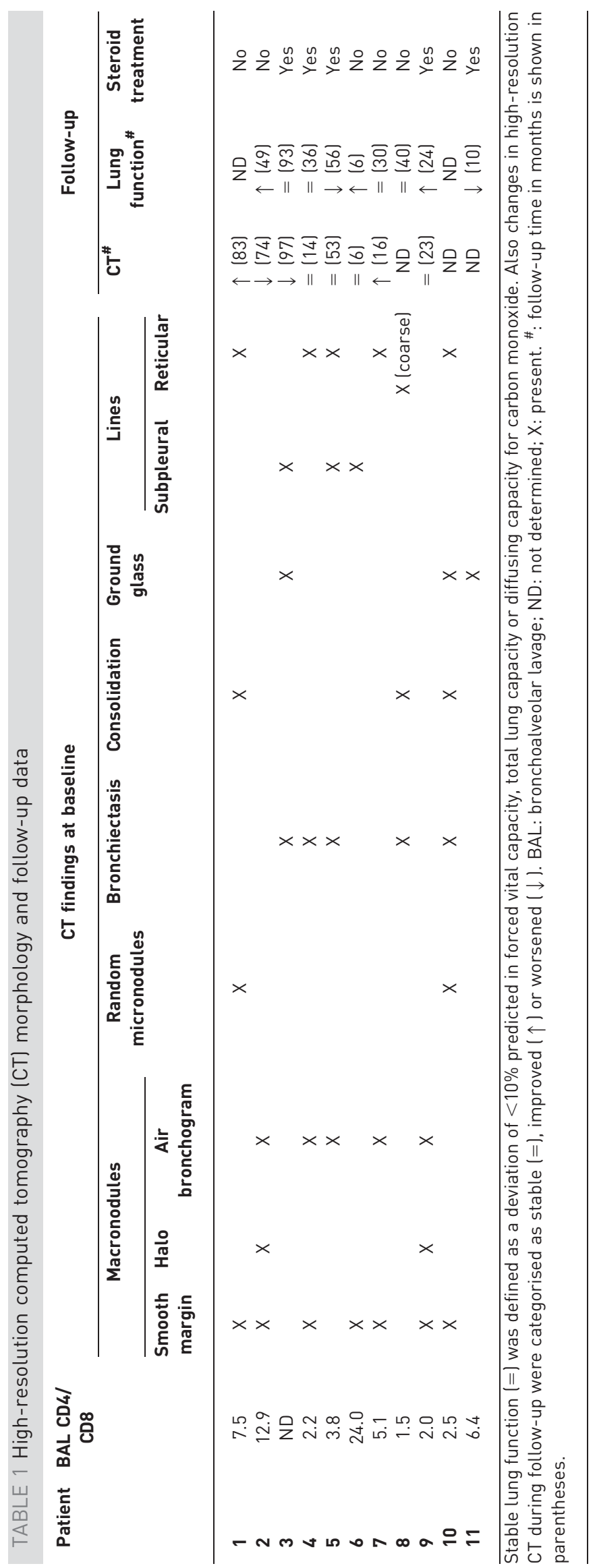


signs and bronchiectasis predominated in CVID. In line with their findings, in our cohort with proven granulomatous disease, the majority of HRCTs demonstrated macronodular lung involvement (eight out of 11 patients). The two patients with micronodular involvement showed a randomly distributed pattern, which also differs to the characteristic perilymphatic distribution in sarcoidosis, as already shown by BOUVRY et al. [1]. Moreover, some patients presented with predominant coarse reticular lines or groundglass lesions. It is intriguing to speculate whether the difference in CD4/CD8 ratio reflects different forms or stages of pulmonary inflammation in CVID, which may correlate to certain computed tomography morphology. This has been previously described in sarcoidosis, where lower CD4/CD8 ratios and increased neutrophils correlated with progressive, fibrotic disease and the need for steroid treatment [3-5]. In our small CVID cohort, lung function and computed tomography findings were mostly stable during follow-up, suggesting that a high CD4/CD8 ratio in granulomatous CVID is associated with a mild and nonprogressive ILD (table 1). Only two patients showed a deterioration of lung function parameters. Interestingly, both of these patients showed rather low CD4/CD8 ratios in comparison to the total study population (2.0 and 3.8).

In conclusion, in addition to the report by BOUVRY et al. [1], we found that in a subgroup of CVID patients with histologically confirmed granuloma formation, BAL cytology resembles sarcoidosis. All patients presented with high BAL lymphocytosis, high CD4/CD8 ratio and the majority with nodular lung disease on computed tomography. Thus, differences in BAL cytology and computed tomography morphology most likely reflect heterogeneity in CVID-associated granulomatous and interstitial lung disease. Our preliminary data suggest a rather favourable prognosis of ILD in this subgroup of CVID with high CD4/CD8 ratio in the BAL. Some of these findings may potentially guide management and predict outcome of these patients, but larger studies are needed to determine diagnostic and prognostic value of specific markers during detailed characterisation of interstitial lung disease in this heterogeneous disease.

○ @ERSpublications

Bronchoalveolar lavage cytology resembles sarcoidosis in a subgroup of granulomatous chronic variable immunodeficiency http://ow.ly/pU6Pl

Florian Kollert ${ }^{1,2}$, Nils Venhoff ${ }^{1,2}$, Sigune Goldacker $^{2}$, Claudia Wehr $^{2}$, Niklas Lützen ${ }^{3}$, Reinhard E. Voll ${ }^{1,2}$, Antje Prasse $^{4,5}$ and Klaus Warnatz ${ }^{1,2,5}$

${ }^{1}$ Centre of Chronic Immunodeficiency, University Medical Centre and University of Freiburg, Freiburg, ${ }^{2}$ Dept of Rheumatology and Clinical Immunology, University Medical Centre Freiburg, Freiburg, ${ }^{3}$ Dept of Radiology, University Medical Centre Freiburg, Freiburg, and ${ }^{4}$ Dept of Pneumology, University Medical Centre Freiburg, Freiburg, Germany.

${ }^{5}$ Both authors contributed equally.

Correspondence: F. Kollert, Dept of Rheumatology and Clinical Immunology, University Medical Centre Freiburg, Hugstetterstr. 55, Freiburg 79106, Germany. E-mail: florian.kollert@uniklinik-freiburg.de

Received: Feb 102013 | Accepted after revision: July 302013

Conflict of interest: Disclosures can be found alongside the online version of this article at www.erj.ersjournals.com

\section{References}

1 Bouvry D, Mouthon L, Brillet PY, et al. Granulomatosis-associated common variable immunodeficiency disorder: a case-control study versus sarcoidosis. Eur Respir J 2013; 41: 115-122.

2 Al-Herz W, Bousfiha A, Casanova JL, et al. Primary immunodeficiency diseases: an update on the classification from the international union of immunological societies expert committee for primary immunodeficiency. Front Immunol 2011; 8: 54.

3 Prasse A, Katic C, Germann M, et al. Phenotyping sarcoidosis from a pulmonary perspective. Am J Respir Crit Care Med 2007; 177: 330-336.

4 Ziegenhagen MW, Rothe ME, Schlaak M, et al. Bronchoalveolar and serological parameters reflecting the severity of sarcoidosis. Eur Respir J 2003; 21: 407-413.

5 Kollert F, Geck B, Suchy R, et al. The impact of gas exchange measurement during exercise in pulmonary sarcoidosis. Respir Med 2010; 105: 122-129.

Eur Respir J 2014; 43: 922-924 | DOI: 10.1183/09031936.00025513 | Copyright @ERS 2014

From the authors:

We would like to thank F. Kollert and co-workers for their interest in our article [1] and their accurate analysis and comments.

Our study was designed to compare interstitial lung disease (ILD) in granulomatosis-associated common variable immunodeficiency disorder (CVID) (ILD/CVID/granulomatous disease) with pulmonary sarcoidosis. 